\title{
The Relationship Between Sarcopenia and Vitamin D Levels in Adults of Different Ethnicities: Findings From the West China Health and Aging Trend Study
}

\section{Xiaoyan Chen}

Zigong Mental Health Center

\section{Lisha Hou}

West China Hospital of Sichuan University

Jirong Yue

West China Hospital of Sichuan University

Yang Wang

West China Hospital of Sichuan University

Xin Xia

West China Hospital of Sichuan University

Birong Dong ( $\boldsymbol{Q}$ birongdong123@outlook.com )

West China Hospital of Sichuan University

Shuyue Luo

West China Hospital of Sichuan University

Xiaolei Liu

West China Hospital of Sichuan University

\section{Research Article}

Keywords: vitamin D, sarcopenia, aging, Western China

Posted Date: February 8th, 2021

DOI: https://doi.org/10.21203/rs.3.rs-154233/v1

License: (c) (1) This work is licensed under a Creative Commons Attribution 4.0 International License. Read Full License 
The relationship between sarcopenia and vitamin D levels in adults of different ethnicities: Findings from the West China Health and Aging Trend study

Running Title: The relationship between sarcopenia and vitamin D Findings from the WCHAT

First author: Shuyue Luo, MD; Address: National Clinical Research Center for

Geriatrics, West China Hospital, Sichuan University, Chengdu, Sichuan Province, China;

Co-first author: Xiaoyan Chen, MD; Address: Zigong Mental Health Center; Zigong, Sichuan Province, China;

Shuyue Luo and Xiaoyan Chen contributed equally to this study and should be considered co-first authors of this paper.

Second author: Lisha Hou, PhD; Address: National Clinical Research Center for Geriatrics, West China Hospital, Sichuan University, Chengdu, Sichuan Province, China;

Third author: Jirong Yue, PhD; Address: National Clinical Research Center for Geriatrics, West China Hospital, Sichuan University, Chengdu, Sichuan Province, China;

Fourth author: Xiaolei Liu, PhD; Address: National Clinical Research Center for Geriatrics, West China Hospital, Sichuan University, Chengdu, Sichuan Province, China;

Fifth author: Yang Wang, MD; Address: National Clinical Research Center for 
Geriatrics, West China Hospital, Sichuan University, Chengdu, Sichuan Province, China;

Sixth author: Xin Xia, MD; Address: National Clinical Research Center for

Geriatrics, West China Hospital, Sichuan University, Chengdu, Sichuan Province, China;

\title{
*Corresponding author:
}

Name: Birong Dong, PhD; Address: National Clinical Research Center for Geriatrics, West China Hospital, Sichuan University, Chengdu, Sichuan Province, China

Email: birongdong123@outlook.com.

Telephone: +8618980601332

\begin{abstract}
Objective: Sarcopenia is a condition associated with progressive loss of skeletal muscle mass and function resulting in substantial negative health outcomes and disability in older adults. The present study was designed to identify risk factors associated with sarcopenia incidence in various ethnic groups in Western China based upon the Asian Working Group on Sarcopenia 2019 (AWGS2019) criteria in various ethnic groups in Western China and to explore the relationship between sarcopenia and vitamin D levels.
\end{abstract}

Study design: A cross-sectional study of individuals that were 50 years of age or older $(n=4236)$ from the West China Health and Aging Trend (WCHAT) study.

Primary outcome and measures: An InBody 770 instrument was used for bioimpedance-based analyses of muscle mass, while a digital grip strength 
dynamometer was used for handgrip strength-based measurements of muscle strength. Physical performance was assessed based upon gait speed over $4 \mathrm{~m}$. Other secondary variables were additionally analyzed as potentially relevant risk factors.

Results: Sarcopenia affected an estimated $22.45 \%$ of studied individuals who were 50 years of age or older, with respective incidence rates in the $<60,60-64,65-79$, and $\geq 80$ age groups of $11.78 \%, 19.44 \%, 32.65 \%$, and $67.97 \%$. Rates in males and females were $26.66 \%$ and $20.05 \%$, respectively. In males, a significant difference in vitamin D levels was detected when comparing individuals with and without sarcopenia, although no such relationship was detected in females. Following adjustment for confounding variables, binary logistic regression analyses revealed that inadequate vitamin $\mathrm{D}$ was able to independently predict sarcopenia risk only in males $(\mathrm{OR}=1.875,95 \% \mathrm{CI}$ : 1.109 $3.169, P=0.019)$.

Conclusions: Among middle-aged and older adults of multiple ethnicities in western China, we found that inadequate vitamin D was an independent predictor of sarcopenia risk specifically in males.

Keywords: vitamin D, sarcopenia, aging, Western China

\section{Introduction}

The definition of sarcopenia was first proposed in 1989 by Irwi Rosenberg[1], and has since undergone multiple updates and improvements. In general, sarcopenia is considered to be an aging-related disease characterized by the gradual loss of muscle mass and functionality [2], resulting in adverse events including falls, disability, reduced quality of life, and an elevated risk of mortality [3, 4]. The etiology of sarcopenia is complex and multifactorial, and greater efforts are required to understand this disease and to raise awareness regarding its severity [5]. The onset of this condition is linked to both contemporaneous risk factors as well as other genetic and lifestyle risk factors encountered throughout the lifespan of a given individual. Relevant risk factors include anorexia, aging and associated changes in hormone levels, bed rest, malnutrition, and a sedentary lifestyle [6]. Age-related sarcopenia incidence has also 
been closely linked to low baseline serum vitamin D levels [7, 8], and the diagnosis of this condition has historically been based upon the European Working Group on Sarcopenia in the Elderly (EWGSOP) or Asian Working Group on Sarcopenia (AWGS2014) diagnostic criteria for sarcopenia. Based upon AWGS2014 diagnostic criteria, sarcopenia is estimated to affect $5.5-25.7 \%$ of individuals in the community, and to affect males more frequently than females [9]. In 2019, AWGS released the latest consensus criteria which included revisions regarding the diagnosis, treatment, and corresponding thresholds associated with sarcoidosis in Asian populations [10]. The impact of these revised AWGS 2019 criteria on studies of sarcopenia incidence and prognosis remains to be established. According to these criteria, the population-adjusted sarcopenia prevalence in Singapore was estimated to be $13.6 \%$ overall $(13.0 \%$ in males, $14.2 \%$ in females), with a higher incidence of $32.2 \%$ in those 60 years of age or older (33.7\% in males, $30.9 \%$ in females) [11]. In addition to being the most populous Asian nation, China has the largest aging population in the world. Western China is a region with populations of multiple ethnic backgrounds, and the association between sarcopenia and vitamin D levels in these populations remains to be defined as per the AWGS2019 diagnostic criteria. Herein, we leveraged cross-sectional data from the West-China Health and Aging Trend (WCHAT) study to conduct a longitudinal multicenter cohort analysis of adults in western China with the goal of evaluating the association between vitamin D levels and sarcopenia as defined according to the AWGS2019 criteria.

\section{Methods}

\section{Study design and patient recruitment}

A cross-sectional survey was conducted to explore the incidence of sarcopenia in middle-aged and older adults in western China and to identify risk factors associated with this condition. This survey employed a multi-stage random cluster sampling approach with a response rate of 50.2\%. Baseline WCHAT study data were collected from July - December 2018 and included individuals from four ethnic backgrounds (Han, Tibetan, Qiang, and Yi) and four provinces (Yunnan, Guizhou, Sichuan, and 
Xinjiang). WHCAT study inclusion was open to individuals who were 50 years of age or older in 2018 and who had resided in the same region for over 36 months. Individuals were excluded from this study if they failed to provide informed consent or had a life expectancy of under six months. Before surveys were conducted, all participants provided written informed to consent. Initially, 7536 community-dwelling individuals meeting study inclusion criteria were recruited, of whom 4433 completed the bioelectrical impedance analysis (BIA), grip strength, and $4 \mathrm{~m}$ habitual walking speed tests necessary to evaluate them for sarcopenia. Patients were excluded from this study if they exhibited vitamin D deficiencies (81), kidney disease (87), mental illness (5), or tumors (24), leaving 4236 patients for our final analyses. The Ethical Review Committee of West China Hospital of Sichuan University approved the present study.

\section{Measures}

All data collection was conducted via face-to-face interviews with individual participants in the four provinces. Interviewers were medical students from West China Clinical Medical College, Sichuan University and had undergone two days of training regarding the appropriate collection of questionnaire data. Anthropometric and BIA measurements were collected by trained technicians. All of the measurements used in this study have been validated in older populations [12]. Demographic factors associated with individual study subjects including age, ethnicity, marital status, smoking history, alcohol consumption history, body mass index (BMI), education, vision, hearing, comorbidities, and sleep were recorded.

\section{Primary outcome}

Sarcopenia incidence was the primary outcome measure of the present study, and was defined as per the AWGS2019 consensus criteria as low muscle mass and low muscle strength or performance [10]. An InBody 770 instrument (BioSpace, Seoul, Korea) was used for BIA measurements of muscle mass, as this approach has been validated in China[13, 14], with low muscle mass being an appendicular skeletal muscle mass(ASM) index in males and females of $<7.0 \mathrm{~kg} / \mathrm{m}^{2}$ and $<5.7 \mathrm{~kg} / \mathrm{m}^{2}$, respectively. 
A myometer (EH101; Camry, Zhongshan, China) was used to test grip strength on two separate occasions, with the maximum value being recorded. Low grip strength in males and females was defined as $<28.0 \mathrm{~kg}$ and $<18.0 \mathrm{~kg}$, respectively, while a low level of physical performance was defined as a gait speed of $<1.0 \mathrm{~m} / \mathrm{s}$. BMI values were calculated based upon participant weight and height. Blood samples were collected from participants in the early morning following an overnight fast, and were used to measure serum hemoglobin, albumin, cholesterol, fasting glucose, vitamin D levels, and additional relevant indicators.

\section{Secondary outcomes}

The 10-item Short Portable Mental Status Questionnaire (SPMSQ)[15] was used to measure cognitive status. An SPMSQ score of 0-2 was indicative of normal cognitive function, while scores of $3+$ indicated cognitive dysfunction. Depressive symptoms were evaluated with the 15-item Geriatric Depression Scale (GDS-15) [16], with scores of $\geq 5$ being considered indicative of depression.

\section{Statistical analysis}

SPSS v 23.0 was used for all statistical testing. All data analyses were stratified according to patient sex. Continuous variables were given as means and standard deviations, while categorical variables were given as frequencies and percentages. Baseline characteristics between patient groups were compared via rank-sum tests and Pearson's chi-square tests, while the association between sarcopenia and vitamin D levels was assessed via a binary logistic regression approach. Odds ratios and 95\% confidence intervals were first calculated with a non-adjusted model, and were then determined using a second model adjusted for age, ethnicity, marital status, smoking history, drinking history, BMI, education level, vision, hearing, comorbidity, and sleep, followed by a third model that further adjusted for cognitive function and depression.

\section{Results}


In total, 4236 participants $\geq 50$-years-old were recruited for this study (1538 males, 2698 females), with their characteristics being compiled in Table 1.

Table 1

Distribution (number with percentage) of participants grouped according to sarcopenic status in classification variables in sex groups

\begin{tabular}{|c|c|c|c|c|c|c|}
\hline \multirow[b]{2}{*}{ Variables } & \multicolumn{3}{|c|}{ Male $(\mathrm{n}=1538)$} & \multicolumn{3}{|c|}{ Female $(n=2698)$} \\
\hline & $\begin{array}{l}\text { Sarcopenic } \\
\qquad(\mathrm{n}=410)\end{array}$ & $\begin{array}{l}\text { Non-sarcopenic } \\
\qquad(\mathrm{n}=1128)\end{array}$ & $P$ & $\begin{array}{l}\text { Sarcopenic } \\
\qquad(\mathrm{n}=541)\end{array}$ & $\begin{array}{l}\text { Non-sarcopenic } \\
\qquad(\mathrm{n}=2157)\end{array}$ & $P$ \\
\hline Age(years),m（iqr） & 67 & 62 & $<0.01$ & 66 & 60 & $<0.01$ \\
\hline$<60, \mathrm{n}(\%)$ & $65(15.85)$ & $428(37.94)$ & & $133(24.58)$ & $1055(48.91)$ & \\
\hline $60-64, \mathrm{n}(\%)$ & $78(19.02)$ & 270(23.94) & & 108(19.96) & $501(23.23)$ & \\
\hline $65-79, \mathrm{n}(\%)$ & $231(56.34)$ & $419(37.15)$ & & $249(46.03)$ & $571(26.47)$ & \\
\hline$>=80, \mathrm{n}(\%)$ & $36(8.79)$ & $11(0.97)$ & & $51(9.43)$ & $30(1.39)$ & \\
\hline Education, $\mathrm{n}(\%)$ & & & $<0.01$ & & & $<0.01$ \\
\hline Elementary school and & $256(6514)$ & $569(5348)$ & & $393(7631)$ & $1376(6709)$ & \\
\hline Middle school & $73(18.58)$ & $266(25)$ & & $80(15.53)$ & $440(21.45)$ & \\
\hline High school and above & $64(16.28)$ & $229(21.52)$ & & $42(8.16)$ & $235(11.46)$ & \\
\hline Marital status, $\mathrm{n}(\%)$ & & & 0.005 & & & $<0.01$ \\
\hline Married & $48(12.21)$ & $80(7.52)$ & & $152(29.51)$ & $356(17.36)$ & \\
\hline Single/Divorced/Widow & $345(87.79)$ & 984(92.48) & & $363(70.49)$ & $1695(82.64)$ & \\
\hline Ethnic, $\mathrm{n}(\%)$ & & & $<0.01$ & & & $<0.01$ \\
\hline
\end{tabular}




$\begin{array}{ccccc}\text { Han } & 201(49.02) & 404(35.82) & 283(52.31) & 947(43.90) \\ \text { Zang } & 117(28.54) & 396(35.11) & 126(23.29) & 548(25.41) \\ \text { Qiang } & 71(17.32) & 276(24.47) & 73(13.49) & 586(27.17) \\ \text { Yi } & 21(5.12) & 52(4.61) & 59(10.91) & 76(3.52)\end{array}$

Comorbidity, $n(\%)$

0.439

0.130

$\begin{array}{lllll}\text { No } & 363(88.54) & 982(87.06) & 457(84.47) & 1901(88.13) \\ \text { Yes } & 47(11.46) & 146(12.94) & 84(15.53) & 256(11.87)\end{array}$

Smoking history, $\mathrm{n}(\%)$

0.047 $<0.01$

$\begin{array}{lll}\text { No } & 202(51.40) & 606(57.22) \\ \text { Yes } & 191(48.60) & 453(42.78)\end{array}$

485(95.28) 2002(97.95)

$24(4.72) \quad 42(2.05)$

Drinking history,n(\%)

0.076

0.284

$\begin{array}{lll}\text { No } & 225(57.25) & 551(52.03) \\ \text { Yes } & 168(42.75) & 508(47.97)\end{array}$

$450(88.24)$

1766(86.44)

60(11.76) 277(13.56)

\section{Cognitive function, $\mathrm{n}(\%)$}

$<0.01$

$<0.01$

Intact

Impaired

Hearing, $n(\%)$

Intact

Impaired

Vision, $\mathrm{n}(\%)$

Intact

Impaired

Sleeping, $n(\%)$
341(86.77) 986(93.28)

$52(13.23) \quad 71(6.72)$

$<0.01$

264(64.39) $881(78.10)$

146(35.61) 247(21.90)

0.014

375(91.46) 1070(94.86)

$35(8.54) \quad 58(5.14)$
$378(74.26)$

131(25.74)

$1738(85.20)$

302(14.80)

$367(67.84) \quad 1801(83.50)$

174(32.16) $\quad 356(16.50)$

289(80.95)

777(84.46)

68(19.05)

143(15.54)
$<0.01$

0.130

0.28
0.110 


\begin{tabular}{ccccccc} 
Well & $219(56.01)$ & $561(52.82)$ & & $280(55.45)$ & $1060(51.48)$ & \\
Poor & $172(43.99)$ & $501(47.18)$ & & $225(44.55)$ & $999(48.52)$ & \\
VitD,ng/mL,mean $( \pm$ SD) & $20.05(6.50)$ & $21.68(6.43)$ & $<0.01$ & $17.58(6.13)$ & $17.92(5.73)$ & 0.2121 \\
BMI,kg/m2,mean( \pm SD) & $22.28(3.06)$ & $26.09(3.47)$ & $<0.01$ & $22.18(3.50)$ & $26.27(3.63)$ & $<0.01$ \\
& 2.50 & 2.37 & & & & \\
GDS score,mean $( \pm$ SD) & $(2.10)$ & $(2.24)$ & 0.322 & $3.11(2.64)$ & $2.70(2.38)$ & $<0.01$ \\
\hline
\end{tabular}

Abbreviations: BMI, body mass index; GDS, geriatric depression scale.

\section{Sarcopenia}

Sarcopenia was found to affect $22.45 \%$ of the overall study population, and to affect $11.78 \%, 19.44 \%, 32.65 \%$, and $67.97 \%$ of individuals aged $<60,60-64,65-79$, and $\geq 80$ respectively. Sarcopenia affected $26.66 \%$ of males in this study, and there were significant differences in age, education, marital status, ethnicity, smoking history, vision, hearing, vitamin D levels, BMI, and cognitive function between males with and without sarcopenia (Table 1). A total of $20.05 \%$ of females in this study were affected by sarcopenia, and there were significant differences in age, education, marital status, ethnicity, smoking history, hearing, BMI, cognitive function, and GDS scores between those females with and without sarcopenia (Table 1).

Vitamin D deficiency was defined by a serum $25(\mathrm{OH}) \mathrm{D}$ level of $20-30 \mathrm{nmol} / \mathrm{L}$, while inadequate vitamin D was defined by serum $25(\mathrm{OH}) \mathrm{D}$ levels $<20 \mathrm{nmol} / \mathrm{L}$. When patients were stratified according to vitamin D levels, we found that sarcopenia prevalence was higher in both males and females with lower levels of serum vitamin D, although this difference was only significant in males (Table 2).

Table 2

The difference of vitamin D between sarcopenia and non-sarcopenia in sex groups

Male

non-sarcopenia

$\mathbf{n}(\%)$
$P$-value

Female

$P$-value

$\begin{array}{cccc}\text { Sarcopenia } & \text { non-sarcopenia } & \text { Sarcopenia } & \text { non-sarcopenia } \\ \mathbf{n}(\%) & \mathbf{n}(\%) & \mathbf{n}(\%) & \mathbf{n}(\%)\end{array}$




\begin{tabular}{|c|c|c|c|c|c|c|}
\hline $\mathrm{VitD}<20 \mathrm{ng} / \mathrm{mL}$ & $218(53.17)$ & $484(42.91)$ & 0.001 & $373(68.95)$ & $1468(68.06)$ & 0.616 \\
\hline $20 \mathrm{ng} / \mathrm{mL} \leqslant \mathrm{VitD}<30 \mathrm{ng} / \mathrm{mL}$ & $157(38.29)$ & $531(47.07)$ & & $155(28.65)$ & $619(28.70)$ & \\
\hline $30 \mathrm{ng} / \mathrm{mL} \leqslant \mathrm{VitD}$ & $35(8.54)$ & $113(10.02)$ & & $13(2.4)$ & $70(3.24)$ & \\
\hline
\end{tabular}

The relationship between vitamin $\mathrm{D}$ and sarcopenia was assessed using three models, with patients with sufficient vitamin D levels serving as controls. Model 1 (non-adjusted) and Model 2 (partially adjusted) exhibited no significant association between vitamin D and sarcopenia in males or females. However, a fully-adjusted Model 3 revealed inadequate vitamin D to be an independent risk factor for sarcopenia incidence in males $(\mathrm{OR}=1.875,95 \% \mathrm{CI}: 1.109-3.169, \mathrm{P}=0.019)$, although no such significant relationship was detected for females (Table 3).

Table 3

The association between vitamin D and sarcopenia in sex groups

\begin{tabular}{|c|c|c|c|c|c|c|c|c|}
\hline Model & Sex & Variables & $\boldsymbol{B}$ & $S E$ & Wald & P-value & OR & $95 \% C I$ \\
\hline \multirow[t]{4}{*}{ Model 1} & males & $20 \mathrm{ng} / \mathrm{mL} \leqslant \mathrm{VitD}<30 \mathrm{ng} / \mathrm{mL}$ & -0.046 & 0.214 & 0.047 & 0.828 & 0.955 & $0.628-1.451$ \\
\hline & & VitD $<20 \mathrm{ng} / \mathrm{mL}$ & 0.374 & 0.21 & 3.181 & 0.074 & 1.454 & $0.964-2.194$ \\
\hline & females & $20 \mathrm{ng} / \mathrm{mL} \leqslant \mathrm{VitD}<30 \mathrm{ng} / \mathrm{mL}$ & 0.299 & 0.315 & 0.9 & 0.343 & 1.348 & $0.727-2.5$ \\
\hline & & VitD $<20 \mathrm{ng} / \mathrm{mL}$ & 0.313 & 0.308 & 1.039 & 0.308 & 1.368 & $0.749-2.5$ \\
\hline \multirow[t]{4}{*}{ Model 2} & males & $20 \mathrm{ng} / \mathrm{mL} \leqslant \mathrm{VitD}<30 \mathrm{ng} / \mathrm{mL}$ & -0.128 & 0.222 & 0.332 & 0.565 & 0.880 & $0.570-1.359$ \\
\hline & & VitD $<20 \mathrm{ng} / \mathrm{mL}$ & 0.386 & 0.218 & 3.133 & 0.077 & 1.471 & $0.959-2.256$ \\
\hline & females & $20 \mathrm{ng} / \mathrm{mL} \leqslant \mathrm{VitD}<30 \mathrm{ng} / \mathrm{mL}$ & 0.620 & 0.462 & 1.801 & 0.18 & 1.860 & $.0751-4.601$ \\
\hline & & VitD $<20 \mathrm{ng} / \mathrm{mL}$ & 0.722 & 0.445 & 2.637 & 0.104 & 2.059 & $0.861-4.924$ \\
\hline \multirow[t]{4}{*}{ Model 3} & males & $20 \mathrm{ng} / \mathrm{mL} \leqslant \mathrm{VitD}<30 \mathrm{ng} / \mathrm{mL}$ & 0.125 & 0.27 & 0.215 & 0.643 & 1.133 & $0.668-1.924$ \\
\hline & & $\mathrm{VitD}<20 \mathrm{ng} / \mathrm{mL}$ & 0.629 & 0.268 & 5.513 & 0.019 & 1.875 & $1.109-3.169$ \\
\hline & females & $20 \mathrm{ng} / \mathrm{mL} \leqslant \mathrm{VitD}<30 \mathrm{ng} / \mathrm{mL}$ & 0.603 & 0.462 & 1.699 & 0.192 & 1.827 & $0.738-4.521$ \\
\hline & & $\mathrm{VitD}<20 \mathrm{ng} / \mathrm{mL}$ & 0.710 & 0.445 & 2.552 & 0.110 & 2.035 & $0.851-4.865$ \\
\hline
\end{tabular}

\section{Discussion}

This study sought to explore the prevalence of sarcopenia as defined according to 
AWGS2019 in a population of individuals 50 years of age and older in Western China, and to assess the relationship between sarcopenia and vitamin D levels. Prior studies have reported sarcopenia incidence rates to vary from $7.8-35.3 \%$ in different Asian countries. Herein, we observed a sarcopenia prevalence of $22.45 \%$, with a higher incidence in males $(26.66 \%)$ relative to females $(20.05 \%)$, and with increasing incidence rates with more advanced age. The prevalence of sarcopenia in our study according to the AWGS2014 diagnostic criteria was previously reported to be 19.31\%[17]. A Singapore cohort study conducted based upon the AWGS2019 diagnostic criteria detected a sarcopenia prevalence of $32.2 \%$ in individuals aged 60 or greater (33.7\% for males, 30.9\% for females) [11]. Differences in these results between studies may be associated with variability with respect to study populations, diagnostic criteria, and instruments used to measure muscle mass.

The association between vitamin D levels and sarcopenia remains controversial. Observational and mechanistic experiments suggest low vitamin D levels to be associated with age-related reductions in muscle mass, indicating that vitamin D supplementation may be an effective approach to preventing or treating sarcopenia. Similarly, we found inadequate vitamin D levels to independently predict sarcopenia risk in males. Mechanistically, vitamin D is a key regulator of the musculoskeletal system, and reduced levels of this vitamin can adversely impact bone health [18]. Vitamin D can control C2C12 skeletal muscle myotube proliferation and differentiation via the mitogen-activated protein kinase signaling pathway [19], and it can further suppress FOXO1 gene expression by controlling calcium channel opening in muscles, thereafter controlling downstream ubiquitin-proteasome system activity [20]. The ubiquitin-proteasome system is, in turn, associated with sarcopenia and skeletal muscle atrophy [21]. The vitamin D receptor(VDR) is expressed in a range of tissues including skeletal muscle in humans, and can bind 1,25,2(OH)D with high affinity, subsequently regulating protein synthesis and the number and volume of type II muscle fibers, thereby controlling skeletal muscle quality and bolstering muscle strength[22]. However, VDR expression is reduced with age [23, 24]. Appropriate vitamin D 
supplementation may control muscle mass and function via VDR-RXR (Nuclear receptor retinoid $\mathrm{X}$ receptor) heterodimer genetic effects and by directly impacting signal transduction pathways[21, 25].

We only found sarcopenia to be significantly associated with vitamin D levels in males. Prior work has suggested that vitamin D is independently linked to decreased strength and loss of muscle mass in elderly populations [26], with more pronounced relationships in men relative to women [26, 27]. One study exploring the relationship between vitamin D deficiencies and gender found sarcopenia risk to be significantly negatively related to serum $25(\mathrm{OH}) \mathrm{D}$ levels in females but not males [28]. These inconsistent findings between groups may be attributable to gender being an important determinant of vitamin D levels[28, 29], to differences in sarcopenia diagnostic criteria and vitamin D deficiency cutoff thresholds, and to varying populations and sample sizes. Future research is necessary to expand our sample size and to validate our results via a cohort study approach.

There are a number of limitations to this study. For one, this was an observational analysis, and it is thus not possible to establish causality. Future large-scale prospective cohort studies will also be required to fully elucidate the differences in these findings between males and females. Second, our measures of cognition, depression, and chronic diseases were based upon questionnaires and should thus be interpreted with caution. Third, this was a study of primarily healthy community-dwelling adults as this was a centralized investigation rather than a household survey, and so the results may not be generalizable to institutionalized or disabled individuals. Despite these limitations, this was a large-scale multi-center study that was able to appropriately adjust for confounding factors in regression analyses, strengthening our overall results.

\section{Conclusion}

In summary, this is one of the only studies to our knowledge to have used the AWGS2019 criteria to gauge sarcopenia prevalence and associated risk factors in Western China. We found sarcopenia to be highly prevalent among older community- 
dwelling adults in this region, and further determined inadequate vitamin D levels to independently predict sarcopenia risk in males. These data thus provide a foundation for interventional efforts to reduce sarcopenia incidence among community residents through exercise- and/or nutrition-based efforts.

\section{Supplementary Information}

The online version contains supplementary material available at

\section{Abbreviations}

VDR: vitamin D receptor; ASM: appendicular skeletal muscle mass; SPMSQ: Short Portable Mental Status Questionnaire; GDS-15: the 15-item Geriatric Depression Scale; BMI: body mass index; BIA: bioelectrical impedance analysis; WCHAT: the West-China Health and Aging Trend; AWGS2019: the Asian Working Group on Sarcopenia 2019; EWGSOP: the European Working Group on Sarcopenia in the Elderly; AWGS2014: Asian Working Group on Sarcopenia 2014.

\section{Acknowledgements}

We thank all personnel for their contribution in the WCHAT study.

\section{Author contributions}

Shuyue Luo and Xiaoyan Chen: Conceptualization, Data curation, Formal analysis, Investigation, Writing - original draft, Writing - review \& editing.

Lisha Hou, Jirong Yue, Xiaolei Liu, Yang Wang, Xin Xia: Investigation, Data curation, Writing review \& editing.

Birong Dong: Conceptualization, Funding acquisition, Investigation, Methodology, Project administration, Supervision, Writing - review \& editing.

All authors were involved in critically revising the manuscript and approved the final manuscript.

\section{Funding}

This work was supported by 1.3.5 project for disciplines of excellence, West China Hospital, Sichuan University (ZY2017201); National Key Research and Development Program of China 
(2017YFC0840100 and 2017YFC0840101); Sichuan Science and Technology Program (2018TJPT0015, 2018JY0094); National Clinical Research Center for Geriatrics, West China Hospital, Sichuan University (Z20191007).

\section{Availability of date and materials}

The datasets used and/or analyzed during the current study available from the corresponding author on reasonable request.

\section{Ethical approval and consent to participate}

The current research was performed in accordance with the Declaration of Helsinki and was approved by the Ethical Review Committee of West China Hospital of Sichuan University with the committee's reference number 2017(445) and the registration number is ChiCTR 1800018895.Before investigation, informed consent was signed and obtained by each participant.

\section{Consent for publication}

Not applicable.

\section{Competing interests}

The authors declare that they have no competing interests.

\section{Authors' information}

National Clinical Research Center for Geriatrics, West China Hospital, Sichuan University, Chengdu, Sichuan Province, China.

\section{References}

1. Rosenberg, I.H., Sarcopenia origins and clinical relevance. American Society for Nutritional Sciences, 1997

2. Cruz-Jentoft, A.J., et al., Sarcopenia: revised European consensus on definition and diagnosis. Age Ageing, 2019. 48(1): p. 16-31

3. Rantanen, T., Muscle strength, disability and mortality. Scand J Med Sci Sportsf,., $f, 2003$

4. Rizzoli, R., et al., Quality of life in sarcopenia and frailty. Calcif Tissue Int, 2013. 93(2): p. 101-20. 
5. Sayer, A.J.C.-J.A.A., Sarcopenia. Lancet, 2019: p. 2636-2646.

6. Marzetti, E., et al., Sarcopenia: an overview. Aging Clin Exp Res, 2017. 29(1): p. 11-17.

7. Hirani, V., et al., Longitudinal Associations Between Vitamin D Metabolites and Sarcopenia in Older Australian men_ The Concord Health and Aging in Men Project. Journals of Gerontology: Medical Sciences, 2018.

8. Anagnostisa, P., et al., Sarcopenia in post-menopausal women_ Is there any role for vitamin D. MATURITAS, 2015.

9. Yoshimura, N., et al., Is osteoporosis a predictor for future sarcopenia or vice versa? Fouryear observations between the second and third ROAD study surveys. Osteoporosis International, 2016. 28(1): p. 189-199.

10. Chen, L.K., et al., Asian Working Group for Sarcopenia: 2019 Consensus Update on Sarcopenia Diagnosis and Treatment. J Am Med Dir Assoc, 2020. 21(3): p. 300-307 e2.

11. Pang, B.W.J., et al., Prevalence and Associated Factors of Sarcopenia in Singaporean Adults-The Yishun Study. J Am Med Dir Assoc, 2020.

12. Henwood, T.R., et al., Assessing sarcopenic prevalence and risk factors in residential aged care: methodology and feasibility. J Cachexia Sarcopenia Muscle, 2014. 5(3): p. 229-36.

13. Wang, H., et al., Estimation of prevalence of sarcopenia by using a new bioelectrical impedance analysis in Chinese community-dwelling elderly people. BMC Geriatr, 2016. 16(1): p. 216.

14. Cruz-Jentoft, A.J., et al., Prevalence of and interventions for sarcopenia in ageing adults: a systematic review. Report of the International Sarcopenia Initiative (EWGSOP and IWGS). Age Ageing, 2014. 43(6): p. 748-59.

15. PFEIFFER, E., A short portable mental status questionnaire for theassessment of organic brain. Journal of the American Geriatrics Society, 1975.

16. Hsu, Y.H., et al., Association of cognitive impairment, depressive symptoms and sarcopenia among healthy older men in the veterans retirement community in southern Taiwan: a cross-sectional study. Geriatr Gerontol Int, 2014. 14 Suppl 1: p. 102-8.

17. Liu, X., et al., Prevalence of sarcopenia in multi ethnics adults and the association with cognitive impairment: findings from West-China health and aging trend study. BMC Geriatr, 2020. 20(1): p. 63.

18. Magdalena Wyskida1, K.W.-T., Jerzy Chudek1, Prevalence and factors promoting the occurrence of vitamin D deficiency in the elderly. Postepy Hig Med Dosw, 2017.

19. Yamazaki, Y., et al., The cathepsin L gene is a direct target of FOXO1 in skeletal muscle. Biochem J, 2010. 427(1): p. 171-8.

20. Hirose, Y., et al., Vitamin D Attenuates FOXO1-Target Atrophy Gene Expression in C2C12 Muscle Cells. J Nutr Sci Vitaminol, 2018.

21. Kamwa, V. and Z.K. Hassan-Smith, The inter-relationship between marginal vitamin D deficiency and muscle. Curr Opin Endocrinol Diabetes Obes, 2019. 26(6): p. 322-328.

22. Shuler, F.D., et al., Sports health benefits of vitamin d. Sports Health, 2012. 4(6): p. 496501.

23. Endo, I., et al., Deletion of vitamin D receptor gene in mice results in abnormal skeletal muscle development with deregulated expression of myoregulatory transcription factors. Endocrinology, 2003. 144(12): p. 5138-44.

24. Campbell, W.W., et al., Dietary protein requirements of younger and older adults. Am J 
Clin Nutr, 2008. 88(5): p. 1322-9.

25. Khammissa, R.A.G., et al., The Biological Activities of Vitamin D and Its Receptor in Relation to Calcium and Bone Homeostasis, Cancer, Immune and Cardiovascular Systems, Skin Biology, and Oral Health. Biomed Res Int, 2018. 2018: p. 9276380.

26. Roth, S.M., et al., Vitamin D receptor genotype is associated with fat-free mass and sarcopenia in elderly men. Journal of Gerontology: BIOLOGICAL SCIENCES, 2004.

27. Granic, A., et al., Vitamin D Status, Muscle Strength and Physical Performance Decline in Very Old Adults: A Prospective Study. Nutrients, 2017. 9(4).

28. Park, S., J.O. Ham, and B.K. Lee, A positive association of vitamin D deficiency and sarcopenia in 50 year old women, but not men. Clin Nutr, 2014. 33(5): p. 900-5.

29. Yan, X., et al., Gender Differences in Vitamin D Status in China. Med Sci Monit, 2019. 25: p. 7094-7099. 


\section{Supplementary Files}

This is a list of supplementary files associated with this preprint. Click to download.

- questionnaire.doc 